\title{
Non-linear Fresnelet approximation for interference term suppression in digital holography
}

\author{
Michael Liebling*, Thierry Blu, Michael Unser \\ Biomedical Imaging Group, Swiss Federal Institute of Technology, Lausanne \\ CH-1015 Lausanne, Switzerland
}

\begin{abstract}
We present a zero-order and twin image elimination algorithm for digital Fresnel holograms that were acquired in an off-axis geometry. These interference terms arise when the digital hologram is reconstructed and corrupt the result. Our algorithm is based on the Fresnelet transform, a wavelet-like transform that uses basis functions tailor-made for digital holography. We show that in the Fresnelet domain, the coefficients associated to the interference terms are separated both spatially and with respect to the frequency bands. We propose a method to suppress them by selectively thresholding the Fresnelet coefficients. Unlike other methods that operate in the Fourier domain and affect the whole spacial domain, our method operates locally in both space and frequency, allowing for a more targeted processing.
\end{abstract}

Keywords: B-splines, Digital holography, Fresnel transform, Fresnelets, Interference, Non-linear approximation.

\section{INTRODUCTION}

When an object is illuminated with a coherent light source, the transmitted or reflected wave carries information on the sample's properties. In the close vicinity of the object, the light intensity is related to its reflectance or attenuation while the phase is related to its thickness. Light sensors, such as CCDs, measure the intensity of the incoming light but are unable to capture its phase. This crucial information is therefore lost. From a mathematical point of view, the measurement of the wave is equivalent to evaluating the squared modulus of the complex scalar field in the acquisition plane, an operation which clearly discards the phase.

Holography overcomes this limitation and makes it possible to record the whole information of the wavefront (amplitude and phase) for later restitution. The hologram measures the intensity of the object wave's interference with a reference wave. In the so-called off-axis geometry, the reference wave and object wave travel in slightly different directions giving rise to interference fringes. To reproduce the object wave, the chemically processed hologram is illuminated with a reconstruction beam which is diffracted. Three diffraction orders may be distinguished: the +1 order which is an exact replica of the object wave, the undiffracted zero-order, and the -1 order.

In digital holography, ${ }^{1-3}$ the photographic plate is replaced by a CCD camera. The hologram is stored in the computer as a digital image and the reconstruction process is carried out by simulating the physical diffraction phenomenon. Since wave propagation can be modeled with good accuracy in the Fresnel régime by the Fresnel transform, it can be easily implemented. Digital holography's advantages are that it is fast (digital holograms may be acquired at video rate) and that it does not involve any chemical processing of the holographic plate or tedious alignment of the reconstruction beam. But most important, quantitative measurements may be performed since the object wave's amplitude and phase are reconstructed digitally. However, since digital recording media have a lower resolution than those used in classical holography, the fringes spacing must be larger in order to be resolved. This means that the reference beam's angle cannot be as high. As a consequence, the three diffracted waves do, at least partially, overlap during reconstruction.

So far, only algorithms have been proposed that either filter the relevant information in the Frequency domain, ${ }^{4-6}$ or, that take advantage of the spatial separation of the different orders after propagation. However, neither approach is completely satisfactory, since either the reconstructed wave's bandwidth or its field of view are drastically limited. Here, we derive a non-linear signal approximation algorithm that takes advantage of the interference terms' separation in both

*E-mail: michael.liebling AT epfl.ch, Tel.: +4121 693 51 43, Fax.: +41 216933701.

Copyright (C)2003 Society of Photo-Optical Instrumentation Engineers.

This paper will be published in the proceedings of the SPIE International Symposium on Optical Science and Technology, Wavelets: Applications in Signal and Image Processing X, 3-8 August 2003, San Diego, CA, USA and is made available as an electronic preprint with permission of SPIE. One print or electronic copy may be made for personal use only. Systematic or multiple reproduction, distribution to multiple locations via electronic or other means, duplication of any material in this paper for a fee or for commercial purposes, or modification of the content of the paper are prohibited. 
frequency and space. To this end, we make use of a recently proposed family of shift invariant, multiresolution basis functions: Fresnelets. ${ }^{7}$

The paper is organized as follows: In Section 2, we review the Fresnel transform and holography. In Section 3, we briefly describe Fresnelets. In Section 4 we propose our new approximation algorithm which we finally illustrate and test on simulation examples in Section 5.

\section{FRESNEL TRANSFORM AND HOLOGRAPHY}

\subsection{Fresnel Transform}

The Fresnel transform $\tilde{f}_{\tau}$ of a function $f \in L_{2}(\mathbb{R})$ with parameter $\tau \in \mathbb{R}_{+}$is defined as the convolution product

$$
\tilde{f}_{\tau}(x)=k_{\tau} * f(x) \text { with } k_{\tau}(x)= \begin{cases}\frac{1}{\tau} \exp \left(i \pi\left(\frac{x}{\tau}\right)^{2}\right) & \tau>0 \\ e^{i \pi / 4} \delta(x) & \tau=0\end{cases}
$$

In 2-D, the Fresnel transform $\tilde{f}_{\tau}$ with parameter $\tau \in \mathbb{R}_{+}$of a function $f \in L_{2}\left(\mathbb{R}^{2}\right)$ is defined as

$$
\tilde{f}_{\tau}(x, y)=f * K_{\tau}(x, y) \text { with } K_{\tau}(x, y)=k_{\tau}(x) k_{\tau}(y)
$$

Up to a complex multiplicative constant, this definition is equivalent to the free-space propagation formula in the Fresnel approximation, which relates the complex values of a propagating wave, measured in two planes perpendicular to the direction of propagation and separated by a distance $d$. Specifically, we have

$$
\begin{aligned}
U_{d}(x, y) & =\frac{e^{i k d}}{i \lambda d} \iint U(\xi, \eta) \exp \left(\frac{i \pi}{\lambda d}\left((\xi-x)^{2}+(\eta-y)^{2}\right)\right) d \xi d \eta \\
& =-i e^{i k d} \tilde{U}_{\tau}(x, y), \quad \tau=\sqrt{\lambda d}
\end{aligned}
$$

where $\lambda$ is the wavelength of the light an $k=2 \pi / \lambda$ its wavenumber.

Three fundamental properties of the Fresnel transform are of particular interest to us, since they give a direct insight on how well the diffraction terms are separated in either space or frequency. First, a modulated signal undergoes a shift after the transform. Let $f \in L_{2}(\mathbb{R})$ and $g(x)=\exp \left(2 i \pi v_{0} x\right) f\left(x+v_{0} \tau^{2} / 2\right)$ be a modulated version of the function. Then its Fresnel transform with parameter $\tau$, is

$$
\tilde{g}_{\tau}(x)=\exp \left(-i \pi v_{0}^{2} \tau^{2}\right) \exp \left(2 i \pi v_{0} x\right) \tilde{f}_{\tau}\left(x-\frac{v_{0} \tau^{2}}{2}\right)
$$

Second, the Fresnel transform is a unitary convolution operator and, as such, the spectrum of the transformed signal remains unchanged. This property may be recognized immediately from the Fresnel operator's frequency response

$$
\hat{k}_{\tau}(v)=e^{i \pi / 4} \exp \left(-i \pi(\tau v)^{2}\right)
$$

where $\left|\hat{k}_{\tau}(v)\right|=1$ implies the spectrum invariance

$$
|\hat{f}(v)|^{2}=\left|\hat{\tilde{f}}_{\tau}(v)\right|^{2} \quad \forall \tau \in \mathbb{R}_{+}
$$

Last, localized features spread out during the propagation process and obey a Heisenberg-like uncertainty principle. ${ }^{7}$ The latter gives a lower bound to the product of a function's variance and that of its transform

$$
\sigma_{f}^{2} \sigma_{\tilde{f}_{\tau}}^{2} \geq \frac{\tau^{4}}{16 \pi^{2}}
$$

For real functions there is also a lower bound on $\sigma_{\tilde{f}_{\tau}} \geq \tau^{2} / 2 \pi$, that is independent of $f$. 


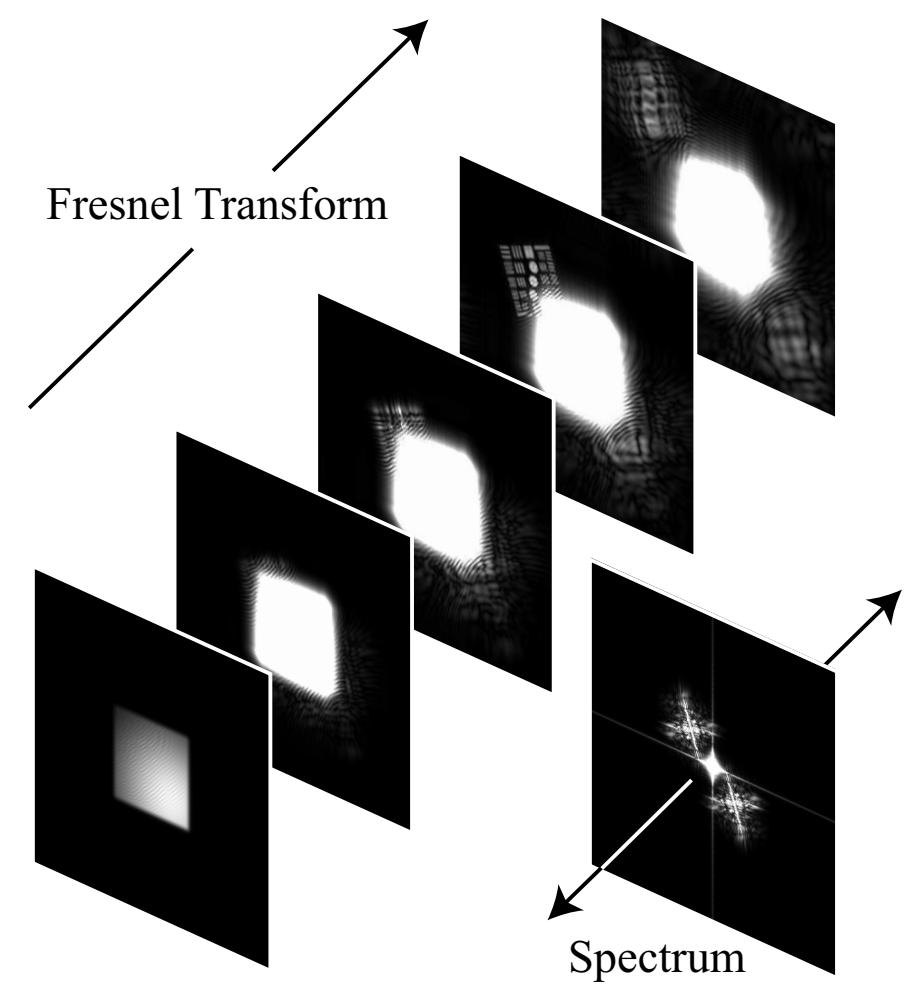

Figure 1. Information repartition in the diffracted wave. Each depth has an associated shift invariant, multiresolution Fresnelet basis. For the in-focus distance, the associated wavelet basis is a standard wavelet basis.

\subsection{Holography}

Information in the hologram plane The hologram measured by the CCD camera, $I(\mathbf{x}) \in \mathbb{R}_{+}$results from the interference, at every location $\mathbf{x}=(x, y)$, of the object wave $\Psi(\mathbf{x}) \in \mathbb{C}$ with a plane reference wave $R(\mathbf{x})=A(\mathbf{x}) \exp \left(i\left(k_{x} x+k_{y} y\right)\right)$ (where $\mathbf{k}=\left(k_{x}, k_{y}, k_{z}\right)$ is the wave vector)

$$
I(\mathbf{x})=|\Psi(\mathbf{x})+R(\mathbf{x})|^{2} .
$$

This equation may be expanded to identify the three interference terms

$$
I(\mathbf{x})=\underbrace{|R(\mathbf{x})|^{2}+|\Psi(\mathbf{x})|^{2}}_{\text {zero-order }}+\underbrace{R^{*}(\mathbf{x}) \Psi(\mathbf{x})}_{+1 \text { order }}+\underbrace{R(\mathbf{x}) \Psi^{*}(\mathbf{x})}_{-1 \text { order }} .
$$

In the hologram plane, they do completely overlap.

Plane wave diffraction by a hologram To reconstruct the object wavefront, we apply a Fresnel transform to the hologram (which is equivalent to physically illuminating the hologram with a plane wave that travels perpendicularly to the hologram). As the propagation distance grows, the unmodulated zero-order stays located in the central part of the image, while the \pm 1 orders move away from the center according to property (5) (see Fig. 1). The higher the modulation frequency (or equivalently, the angle between the reference and the object wave), the larger the separation. Because the acquisition device's sampling step remains large, the modulation frequency is limited, as well as the angle between the object and the reference wave. Therefore, the spatial separation between the different orders is limited and they do, at least partially, overlap.

Moreover, the uncertainty relation on the Fresnel transform implies a broadening of the zero and -1 orders as the distance increases (see Fig. 2). In contrast, the +1 order's support first shrinks until the original image-hologram distance is reached and starts broadening again for larger distances. 


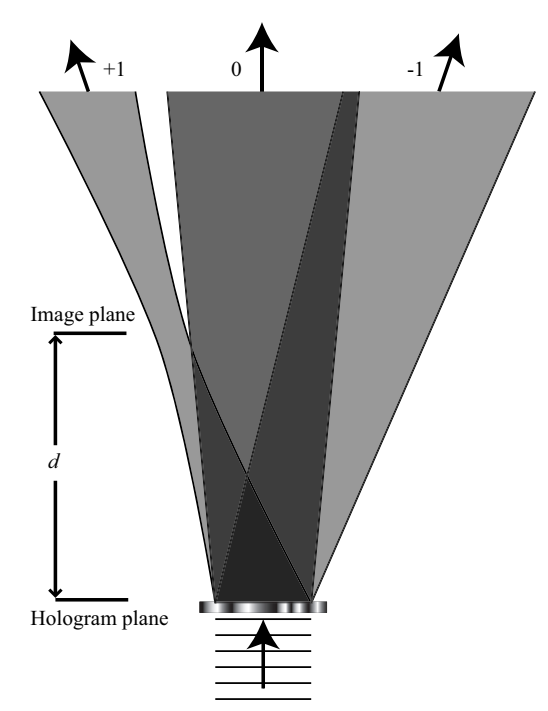

Figure 2. Support broadening of the wave diffracted by an off-axis Fresnel hologram.

Frequency content The three terms have their energy clearly separated in the frequency domain and located around their respective modulation frequency. This property was early recognized to be of use in digital holography, since several algorithms that keep only the relevant frequency information $(+1$ order $)$ and discard the rest via a bandpass filtering procedure have been proposed. Their implementation can be carried out in either the spatial ${ }^{4,6}$ or frequency domain. ${ }^{5}$ All these filtering procedures are linear. However, since they limit the spectral content of the image to reconstruct, details are lost. Moreover, since such filtering procedures are non-local, the whole field of view is affected.

Interestingly, property (7) implies that the spectrum of the diffracted wave at any distance from the hologram remains unchanged (see Fig. 1). This means, that the filtering may be equivalently performed at any distance.

\section{FRESNELETS}

Fresnelet bases are wavelet bases that have undergone a Fresnel transform. We focus on Fresnelets associated with Bspline wavelets, since their expression may be derived in both frequency and space. ${ }^{7}$ They have many desirable properties required for the digital processing of holograms: for example, they tend to be optimal with respect to the spatial energy spreading as they can be shown to converge to Gabor functions. ${ }^{7}$ The construction is based on the definition of the Fresnel spline, or F-spline of degree $n \in \mathbb{N}$ and parameter $\tau \in \mathbb{R}_{+}$, denoted $\tilde{\beta}_{\tau}^{n}(x)$, that is the Fresnel transform with parameter $\tau$ of a B-spline $\beta^{n}(x)$ of degree $n$

$$
\tilde{\beta}_{\tau}^{n}(x)=\left(\beta^{n} * k_{\tau}\right)(x) .
$$

The generating functions are then constructed as linear combinations of F-splines

$$
\tilde{\Psi}_{\tau / 2}^{n}\left(\frac{x}{2}\right)=\sum_{k} g(k) \tilde{\beta}_{\tau}^{n}(x-k)
$$

and are entirely specified from the sequence $g(k)$. They correspond to the general family of semi-orthogonal spline wavelets of the form

$$
\psi^{n}\left(\frac{x}{2}\right)=\sum_{k} g(k) \beta^{n}(x-k)
$$

The transformed basis functions are shift-invariant on a level-by-level basis but their multiresolution properties are governed by the special form that the dilation operator takes in the Fresnel domain. In our case, given that the wavelength is fixed, the parameter $\tau=\sqrt{\lambda d}$ only depends on the depth $d$ of the propagation. For each depth there is an associated basis. From now on, we only consider orthonormal Fresnelet bases of $L_{2}\left(\mathbb{R}^{2}\right)$, denoted

$$
\left\{\tilde{\psi}_{\tau, j, \mathbf{k}}^{n}\right\}_{j \in \mathbb{Z}, \mathbf{k} \in \mathbb{Z}^{2}}, \quad \tilde{\psi}_{\tau, j, \mathbf{k}}^{n}(\mathbf{x})=\frac{1}{\sqrt{2^{j}}} \tilde{\psi}_{\tau / 2^{j}}^{n}\left(\frac{\mathbf{x}}{2^{j}}-\mathbf{k}\right)
$$




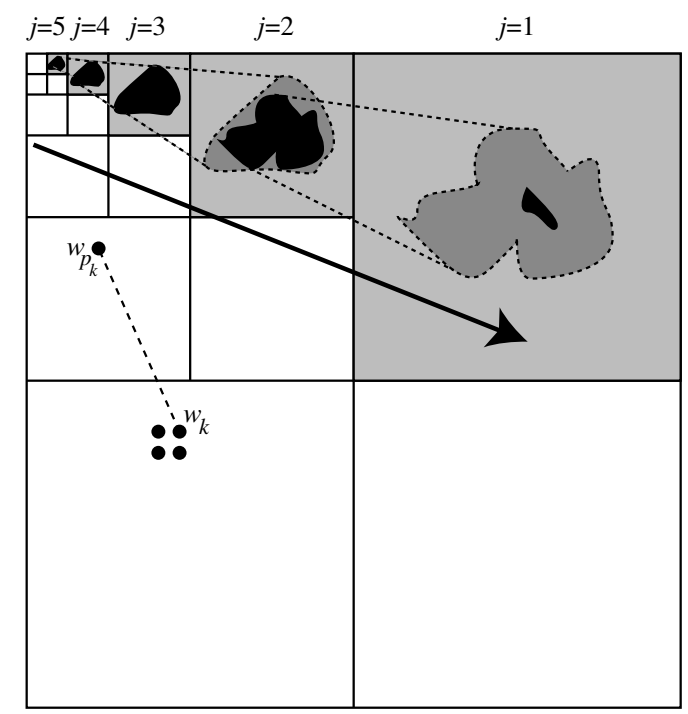

Figure 3. Schematical representation of the hierarchical thresholding algorithm.

For a given setup, a single set of coefficients may be used to generate the diffracted wave at any depth, simply by replacing the basis functions in the expansion

$$
\tilde{f}_{\tau}(\mathbf{x})=\sum_{j \in \mathbb{Z}} \sum_{\mathbf{k} \in \mathbb{Z}^{2}} c_{j, \mathbf{k}} \tilde{\Psi}_{\tau, j, \mathbf{k}}^{n}(\mathbf{x})
$$

with those associated to a different depth. In our case, the diffracting wave in the hologram plane is given by $I(\mathbf{x})$, the coefficients are obtained by computing the inner products

$$
c_{j, \mathbf{k}}=\left\langle I, \tilde{\psi}_{\tau, j, \mathbf{k}}^{n}\right\rangle
$$

\section{ALGORITHM}

We now propose an algorithm that selectively suppresses the Fresnelet coefficients in order to keep only coefficient whose energy is mainly related to the +1 order. It is a fully automatic two-step process. First, we suppress the zero-order and second, the -1 order.

Zero-order suppression The first step consists in the computation of the hologram's Fresnelets coefficients (14), where the parameter $\tau=\sqrt{\lambda d}$ must be adjusted properly. This not only yields a decomposition of the information in several frequency bands, but also in terms of their spatial distribution within the frequency bands. The energy that is associated to the (unmodulated) zero-order is mainly concentrated at low frequencies. The algorithm proceeds from coarse to fine: a threshold value $t_{j}$ is associated to every frequency band $j$. The parent coefficient of $w_{k}$ is denoted $w_{p_{k}}$ (Fig. 3). The new coefficients $w_{k}^{\prime}$ are computed at the coarsest scale $j=J$ as

$$
w_{k}^{\prime}= \begin{cases}w_{k} & \text { if }\left|w_{k}\right|<t_{J} \\ 0 & \text { if }\left|w_{k}\right| \geq t_{J}\end{cases}
$$

and for the finer scales $j<J$

$$
w_{k}^{\prime}= \begin{cases}w_{k} & \text { if }\left|w_{k}\right|<t_{j} \\ 0 & \text { if }\left|w_{k}\right| \geq t_{j} \text { and }\left|w_{p_{k}}\right| \geq t_{j+1} .\end{cases}
$$

Unlike denoising algorithms that set low energy coefficients to zero, our method eliminates high energy coefficients. The test on the parent coefficient ensures that high frequency coefficients are only removed in regions that are corrupted by the zero-order. The signal is reconstructed with Fresnelets of parameter $\tau=0$ which yield a reconstruction with the real image $(+1$ order) at proper focus and the zero-order suppressed. 


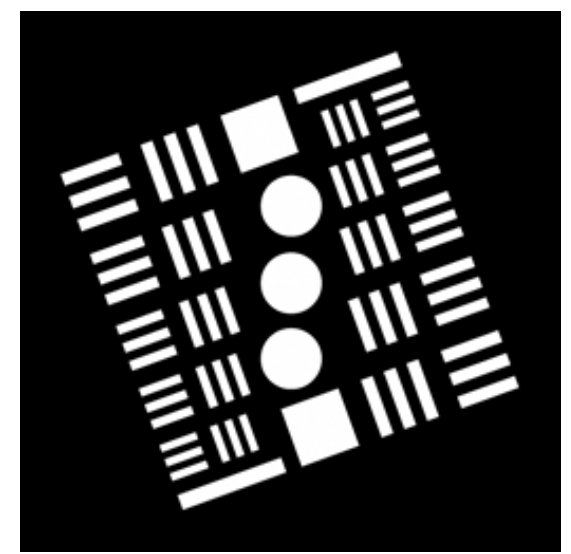

(a)

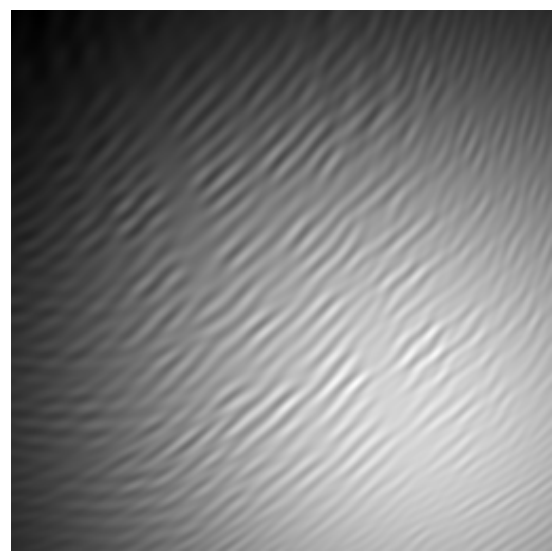

(b)

Figure 4. (a) Amplitude of test target. (256 $\times 256$ pixels, $T=10 \mu \mathrm{m}, d=0.25 \mathrm{~m}, \lambda=632.8 \mathrm{~nm})$ (b) simulated hologram

Minus 1 order suppression Since in the Fresnelet domain, information located around a particular frequency cannot be distinguished from that lying around the opposite sign frequency, and since the +1 and -1 order are indeed located at opposite frequencies, a second step is required to suppress the -1 order. We start by a pointwise multiplication of the wave obtained in the first step, with a digital wave of the form $R^{*}(\mathbf{x})=\exp \left(-i\left(k_{x} x+k_{y} y\right)\right)$, the complex conjugate of the reference wave. This modulation shifts the frequencies such that the -1 order is located around the frequency origin. We then apply a Fresnelet transform with parameter $\tau=0$ before going through the same thresholding scheme as in the first step, but with new thresholding values. After inverse transforming the coefficients and (de)modulating the result using a digital wave of the form $R^{\prime}(\mathbf{x})=\exp \left(2 i\left(k_{x} x+k_{y} y\right)\right)$, we obtain a reconstruction that is free of interference terms.

\section{RESULTS}

A hologram was obtained by simulating the propagation of the wave reflected by a test target by a procedure described elsewhere. ${ }^{7}$ We have chosen the following values for the various parameters: $T=10 \mu \mathrm{m}$ (camera's sampling step), $d=0.25 \mathrm{~m}$ (object-camera distance), $\lambda=632.8 \mathrm{~nm}$ (light wavelength). The angle between the reference wavevector and the normal to the CCD plane was set to $0.45^{\circ}$. The reference wave's intensity profile is Gaussian. The test target and the simulated hologram are shown in Fig. 4.

In Fig. 5(a), we show the wave diffracted by the hologram in the image plane without any interference term suppression scheme applied. The field of view is limited because of the zero-order overlap. A filtering scheme that keeps only a circular frequency band around the +1 order term was used to obtain Fig. 5(b). Finally, in Fig. 5(c), we show the reconstruction with the proposed algorithm. High frequency features are well preserved in regions where the zero-order does not overlap, such as the bars in the upper right. By contrast, the same bars are completely blurred in the bandpass filtering approach. The two approaches behave similarly in regions were the zero-order overlaps. The wavelet-based approach thus only removes high frequency information in regions already corrupted by the zero-order but keeps it intact in other regions.

\section{CONCLUSION}

We have proposed a zero and -1 order term suppression algorithm for digital hologram reconstruction. It takes advantage of the information distribution of the different diffraction terms in both frequency and space. This is made possible by the use of the Fresnelet transform which has the ability to separate the information in the hologram accordingly. Unlike algorithms that are based on a bandpass filtering of the hologram, high frequency features that would normally get lost over the whole field of view are only suppressed where necessary. 


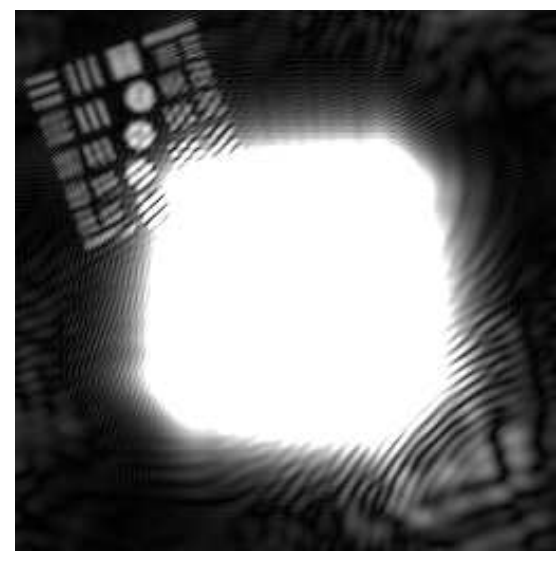

(a)

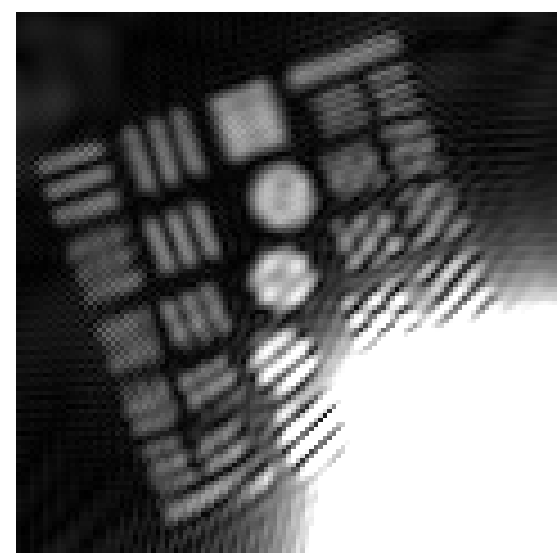

(d)

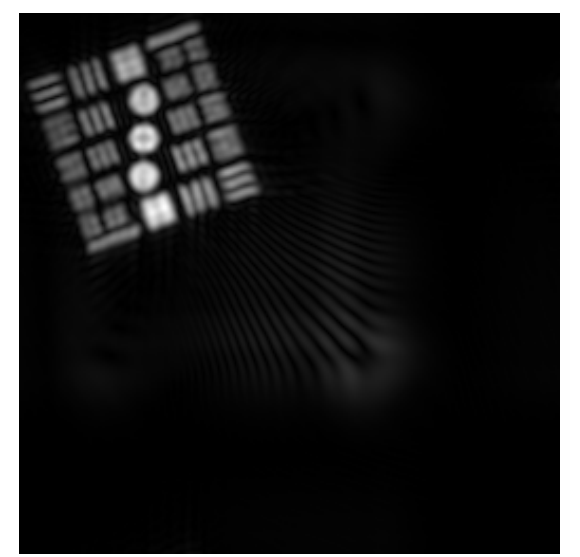

(b)

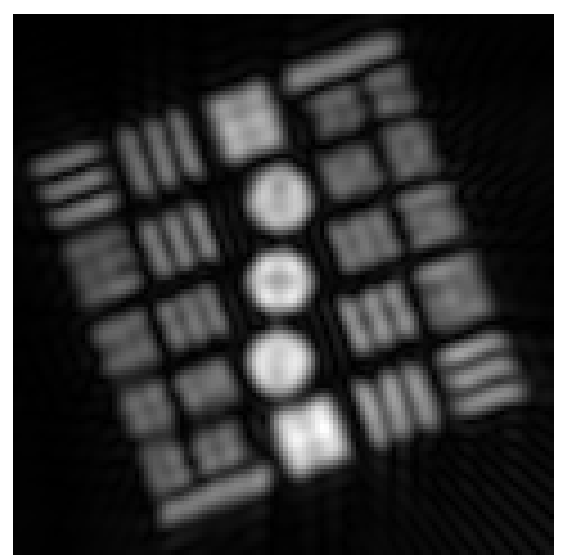

(e)

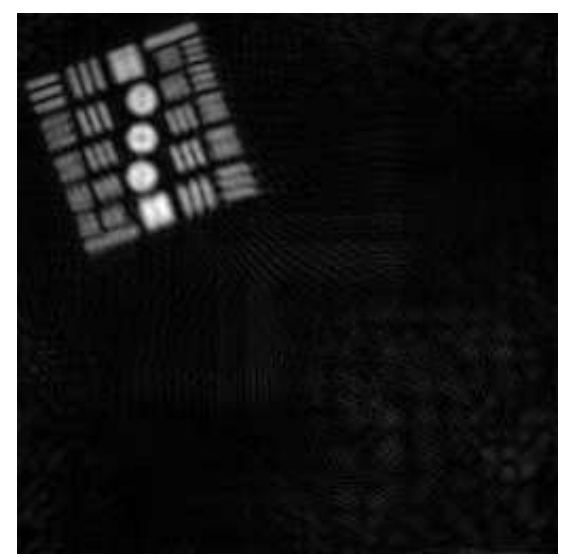

(c)

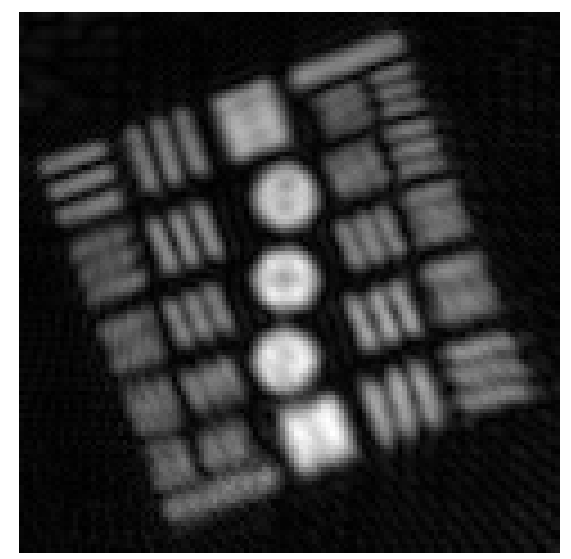

(f)

Figure 5. Reconstructed amplitude: (a) without filter, (b) with frequency filter, (c) with wavelet threshold. (d), (e), (f) detail images.

\section{REFERENCES}

1. J. W. Goodman and R. W. Lawrence, "Digital image formation from electronically detected holograms," Appl. Phys. Lett. 11, pp. 77-79, Aug. 1967.

2. M. A. Kronrod, N. S. Merzlyakov, and L. Yaroslavskii, "Reconstruction of a hologram with a computer," Sov. Phys. Tech. Phys. 17, pp. 333-334, Aug. 1972.

3. E. Cuche, F. Bevilacqua, and C. Depeursinge, "Digital holography for quantitative phase-contrast imaging," Opt. Lett. 24, pp. 291-293, Mar. 1999.

4. T. M. Kreis and W. P. O. Jüptner, "Suppression of the de term in digital holography," Opt. Eng. 36, pp. 2357-60, Aug. 1997.

5. E. Cuche, P. Marquet, and C. Depeursinge, "Spatial filtering for zero-order and twin-image elimination in digital off-axis holography," Appl. Opt. 39, pp. 4070-4075, Aug. 2000.

6. C. Liu, Y. Li, X. Cheng, Z. Liu, F. Bo, and J. Zhu, "Elimination of zero-order diffraction in digital holography," Opt. Eng. 41, pp. 2434-2437, Oct. 2002.

7. M. Liebling, T. Blu, and M. Unser, "Fresnelets: New multiresolution wavelet bases for digital holography," IEEE Trans. Image Proc. 12, pp. 29-43, Jan. 2003. 